\title{
Detail, dynamics and depth: useful correctives for some current research trends
}

\author{
Barnaby Nelson, Jessica A. Hartmann and Josef Parnas
}

\section{Summary}

Several research trends in contemporary psychiatry would benefit from greater emphasis on detailed assessment, modelling dynamic change, and micro-level analysis. This may assist with clarifying nosological and pathoaetiological issues. We make this case by referring to three areas: psychopathology and nosology; prediction research; and 'big N' data sets.

\section{Declaration of interest}

None.

\section{Copyright and usage}

(c) The Royal College of Psychiatrists 2018.
Barnaby Nelson is a clinical psychologist and Professor at Orygen, The National Centre of Excellence in Youth Mental Health, Melbourne. His research focuses mainly on prediction and intervention for early stages of psychotic disorder.

Josef Parnas is a professor of Psychiatry at the University of Copenhagen, Denmark. His research interest is in the domains of pathogenesis and phenomenology of schizophrenia, classification and general psychopathology.

Jessica Hartmann is a McKenzie Postdoctoral Research Fellow at the University of Melbourne and Orygen, the National Centre of Excellence in Youth Mental Health. Her research focuses on at risk mental states and onset of disorder from a transdiagnostic and dynamic perspective.

In this editorial, we argue that a number of tools used in contemporary psychiatric research, particularly in psychopathological assessment, are too blunt. The field would benefit from a greater emphasis on sharper, more detailed assessment and analysis; where there is great surface area, further depth could be introduced; where the 'macro' abounds, let us not forget the value of the 'micro'. This would enrich, balance and provide a corrective to several research trends, and possibly clarify nosological and pathoaetiological issues. We make this case by referring to three areas: psychopathology and nosology, prediction research and 'big N' data-sets.

\section{Psychopathology and nosology}

Much recent work has questioned traditional diagnostic categories ${ }^{1}$ and moved to identify domains of psychopathology and dimensions/continua that cut across diagnostic boundaries. ${ }^{2}$ This can be seen in the checklist- or atomistic-type approach to measuring signs and symptoms, ${ }^{3}$ which have found significant overlap in psychotic and other symptoms across disorders (e.g. between schizophrenia and bipolar disorder ${ }^{4}$ and between psychotic disorders and borderline personality disorder ${ }^{5}$ ), as well as significant presence of 'symptoms' in general community (nonpatient) samples. ${ }^{2}$ The National Institute of Mental Health Research Domain Criteria signal a frustration with the DSM phenotypebased classification system and represent an attempt to base psychiatric nosology on neuroscience and behavioural science instead of descriptive phenomenology. ${ }^{6}$ These approaches promote the idea that phenotypes are far more overlapping and indistinct than traditionally thought, and that psychopathological domains can be measured quickly and simply in order to map on to the 'real' underlying pathology of neurobiological dysfunction. Just as an individual can be genotyped by running a DNA sequence with biological assays, their phenotype can be characterised through efficient (ideally self-report) measures that operationalise the array of psychiatric symptoms and signs.

However, part of the frustration with phenotype-based classification and the perceived roadblock that it has introduced to research progress may be attributable not to phenotype-based classification per se, but rather to the oversimplified and broad nature of contemporary psychopathological description present in DSM-III onwards and many of the instruments used to measure psychopathology in research studies. ${ }^{7}$ What is required is perhaps not so much a discarding or downgrading of the centrality of phenotypes, but rather a resuscitation and refinement of psychopathological understanding and assessment. A deeper and more fine-grained approach to psychopathology reveals important differences that are blurred in many current approaches, and certainly in most of the measurement instruments used to generate phenotypic data. Take the example of anxiety. Generalised anxiety ('excessive worry about aspects of quotidian life') related possibly to neurotic personality features is quite different from the anxiety that a person with schizophrenia might present with concerning how stable and reliable the existence of the world and other people are and a pervasive sense of being exposed ('ontological anxiety'). However, without some degree of probing of the immediate surface complaint and a sense of its psychopathological context, the distinction will not be apparent and will simply be scored as 'anxious' on a symptom checklist. Similar statements could be made about the phenomenon of selfreference. ${ }^{8}$ Although these types of psychopathology might all be four-legged animals, four-legged animals vary a great deal and you mistake a rhinoceros for a pig at your own peril. Kendler ${ }^{9}$ makes the point that in some contexts, such as a busy emergency department situation, straightforward and blunt checklist-type questioning may be good enough. Of course, this is correct and pragmatic decision-making is required in day-to-day clinical work. However, the psychopathological distinctions flagged above are not just indulgent niceties that can be used to show off clinical skill or insight - they might be important markers of different types of disorders (nosology) and related psychological/neurocognitive/neurobiological processes, and therefore failing to detect such distinctions (in fact, as Andreasen ${ }^{10}$ and Kendler ${ }^{9}$ warn, losing the skill required to detect such differences) is a rate-limiting factor in aetiological research.

As well as revealing important differences between mental disorders (differing phenotypes), a detailed approach also reveals 
significant qualitative differences between experiences/symptoms thought to exist on an illness continuum. Self-reported 'psychoticlike experiences' have been found to show very poor correlation with more fine-grained, clinician-assessed 'attenuated psychotic symptoms. ${ }^{11}$ Measured quantitatively on yes/no self-report instruments, nonclinical samples may show ratings not radically dissimilar to ratings in patient samples. However, this may mask dramatic qualitative differences between the experiences/symptoms being endorsed. Stanghellini and colleagues ${ }^{12}$ showed dramatic qualitative differences between hallucinatory experiences in a nonclinical sample and those in a schizophrenia sample. The same positively endorsed Revised Hallucinations Scale items reflected quite different experiences in the two groups. For example, the nonclinical sample prototypically endorsed the item 'I have had the experience of hearing a person's voice and then found that there was no one there' for run-of-the-mill perceptual disturbances (e.g. 'When I'm at home studying, I heard my mother's voice calling me. She lives with me and calls me often, and this might make me think that I hear her calling me when she really is not'), whereas the clinical sample endorsed the item for auditory verbal hallucinations (e.g. 'I hear a voice that asks me to do something or tells me something about someone'). Even putting to one side the issue of how individual symptoms relate to each other and the larger Gestalt of clinical presentation, ${ }^{8}$ declaring a smooth phenomenological continuity for such experiences/symptoms from health to illness is clearly problematic. The view that they are the same type of thing (reflected in the use of the term 'psychotic' for both sets of experiences) but just set at different amplitudes does not square with a more detailed analysis.

\section{Prediction research}

Recent years have also seen an increased focus on attempts to model and predict which high-risk individuals will progress to full-threshold disorder. Psychiatric prediction research, particularly in the area of psychosis prediction, has shown an over-reliance on one-off sampling of cross-sectional data (i.e. a snapshot of clinical state and other risk markers). Predictive models, even if multi-modal in nature, ${ }^{13}$ are limited to a single point in time (study entry or clinic presentation). We have recently argued that predictive modelling may benefit from moving from this convenient but rather static approach to taking dynamic, time-dependent changes into account. Crossdisciplinary approaches to complex system structures and changes (as seen in ecology or economics), such as dynamical systems theory, network theory, instability mechanisms, chaos theory and catastrophe theory, offer useful models that can be applied to the emergence (or decline) of psychopathology. This type of predictive modelling requires repeat longitudinal assessment of relevant variables through either, or a combination of, micro-level (momentary, day-to-day) and macro-level (months, years) assessments. This type of prediction research may more accurately model the highly dynamic nature of psychopathology and system change, as well as have treatment implications, such as introducing a means of identifying critical periods of risk for mental state deterioration. Empirical work adopting this dynamic approach has already shown significant promise ${ }^{14-17}$ and may be particularly relevant to individual-level, 'precision' medicine/prediction. ${ }^{14}$

\section{Big 'N' data}

Finally, much recent research has focused on 'big N' data-sets: multisite studies, data linkage studies, consortia-based research, automatic data collection of digital behaviour, etc. This is clearly of great advantage when it comes to increasing statistical power and addressing broad research questions. However, we also need to be mindful of the limitations of this approach. Such studies can cover a large terrain, but do not necessarily see this terrain in great detail, therefore limiting the types of questions or issues that can be addressed. Take the example of 'digital phenotyping' - that is, measuring behaviour from smartphone sensors, keyboard interaction and various features of voice and speech. ${ }^{18}$ Although this clearly has potential for efficient and large-scale data collection and potential accessibility as an intervention (but keep in mind that there is a certain bias against low- and middle-income countries and more economically deprived people within high-income countries in these digital-based approaches), it also has the potential to make the superficiality of psychopathological assessment flagged above even more superficial. Although the use of technology could assist with characterising certain elements of the individual as a complex system (see above), automatic detection tools (e.g. location mapping, type and degree of activity, level of social engagement, logons on social media and frequency of tweeting) provide a blunt behavioural proxy of mental state and are a profoundly anaemic approach to understanding psychopathology and nosology. Rather than confronting the need for more nuanced and sophisticated approaches to understanding psychopathology (i.e. defining the phenotype), the digital approach just pushes the problem one step further away as well as appealing to the field's longstanding search for methods that appear behavioural and objective. This approach, in our view, is more likely to be a convenient adjunct to phenomenologically informed clinical interviewing ${ }^{19}$ rather than offering a breakthrough on issues of diagnosis or prediction research.

Clearly, the concepts and methods one adopts are dependent on the clinical context and research question; for some situations, the concepts/methods flagged above will be adequate. However, overall these trends indicate a field that is prioritising a broad, sweeping view of psychopathology and ease of data acquisition over detailed examination of clinical phenomena, which may ultimately distort our view of the 'object' of psychiatry (an individual's altered experience, expression and existence associated with suffering in self and/or others ${ }^{3}$ ). They indicate a lack of detailed phenomenology (a 'phenophobia' ${ }^{20}$ ) in understanding psychopathology and nosology, an over-reliance on static rather than dynamic forms of prediction, and aspirations towards big ' $\mathrm{N}$ ' studies with superficial data. Rather than make these approaches into golden calves, it would be more useful to upgrade our clinical lens to a finergrained resolution, enhance predictive models with assessment of temporal dynamics and supplement large data-sets with assessments of depth that are not subjectivity averse.

Barnaby Nelson, PhD, Jessica A. Hartmann, PhD, Orygen, the National Centre of Excellence in Youth Mental Health, Australia, and Centre for Youth Mental Health, University of Melbourne, Australia; Josef Parnas, MD, Psychiatric Center Hvidovre \& Center for Subjectivity Research, University of Copenhagen, Denmark.

Correspondence: Barnaby Nelson, Orygen, 35 Poplar Rd (Locked Bag 10), Parkville, Victoria 3052, Australia. Email: Barnaby.nelson@orygen.org.au

First received 13 Nov 2017, accepted 15 Feb 2018

\section{References}

1 Guloksuv S, van OS J. The slow death of the concept of schizophrenia and the painful birth of the psychosis spectrum. Psychol Med 2018; 48(2): 229-244.

2 van OS J, Reininghaus U. Psychosis as a transdiagnostic and extended phenotype in the general population. World Psychiatry 2016; 15(2): 118-24.

3 Parnas J, Bovet P. Psychiatry made easy: operation(al)ism and some of its consequences. In Philosophical Issues in Psychiatry III: The Nature and Sources 
of Historical Change (eds K Kendler, J Parnas): 191-212. Oxford University Press, 2017.

4 Thaker GK. Boundaries of the psychosis phenotype. Schizophr Bull 2012; 38(2): 205-6.

5 Schroeder $\mathrm{K}$, Fisher HL, Schafer I. Psychotic symptoms in patients with borderline personality disorder: prevalence and clinical management. Curr Opin Psychiatry 2013; 26(1): 113-9.

6 Cuthbert BN. The RDoC framework: facilitating transition from ICD/DSM to dimensional approaches that integrate neuroscience and psychopathology. World Psychiatry 2014; 13(1): 28-35.

7 Parnas J. The RDoC program: psychiatry without psyche? World Psychiatry 2014; 13(1): 46-7.

8 Parnas J, Henriksen MG. Epistemological error and the illusion of phenomenological continuity. World Psychiatry 2016; 15(2): 126-7.

9 Kendler KS. Introduction to the ontology and epistemology of symptoms: the case of auditory verbal hallucinations in schizophrenia. In Philosophical Issues in Psychiatry IV: Classification of Psychiatric Illness (eds KS Kendler, J Parnas): 197-200. Oxford University Press, 2017.

10 Andreasen NC. DSM and the death of phenomenology in America: an example of unintended consequences. Schizophr Bull 2007; 33(1): 108-12.

11 Schultze-Lutter F, Renner F, Paruch J, Julkowski D, Klosterkotter J, Ruhrmann S Self-reported psychotic-like experiences are a poor estimate of clinician-rated attenuated and frank delusions and hallucinations. Psychopathology 2014; 47 (3): 194-201.
12 Stanghellini G, Langer Al, Ambrosini A, Cangas AJ. Quality of hallucinatory experiences: differences between a clinical and a non-clinical sample. World Psychiatry 2012; 11(2): 110-3.

13 Nieman DH, Ruhrmann S, Dragt S, Soen F, van Tricht MJ, Koelman JH, et al. Psychosis prediction: stratification of risk estimation with information-processing and premorbid functioning variables. Schizophr Bull 2014; 40(6): 1482-90.

14 Wichers M, Groot PC, Psychosystems ESMGEWSG. Critical slowing down as a personalized early warning signal for depression. Psychother Psychosom 2016; 85(2): 114-6.

15 Wichers M, Wigman JTW, Myin-Germeys I. Micro-level affect dynamics in psychopathology viewed from complex dynamical system theory. Emot Rev 2015: 7(4): 362-7.

16 Yuen HP, Mackinnon A, Nelson B. A new method for analyzing transition to psychosis: joint modeling of time-to-event outcome with time-dependent predictors. Int J Methods Psychiatr Res 2017; e1588.

17 van de Leemput IA, Wichers M, Cramer AO, Borsboom D, TuerlinckX F, Kuppens $P$, et al. Critical slowing down as early warning for the onset and termination of depression. Proc Natl Acad Sci USA 2014; 111(1): 87-92.

18 Insel TR. Digital phenotyping: technology for a new science of behavior. JAMA 2017: 318(13): 1215-6

19 Nordgaard J, Sass LA, Parnas J. The psychiatric interview: validity, structure and subjectivity. Eur Arch Psychiatry Clin Neurosci 2013; 263(4): 353-64.

20 Parnas J. Delusions, epistemology and phenophobia. World Psychiatry 2015; 14 (2): 174-5. 